\title{
Numerical Studies of Flow Past Two Side-by-Side Circular Cylinders
}

\author{
J. Shao, C. Zhang
}

Department of Mechanical and Materials Engineering, The University of Western Ontario, London, Ontario, Canada N6A $5 B 9$

Email: czhang@eng.uwo.ca

\begin{abstract}
Multiple circular cylindrical configurations are widely used in engineering applications. The fluid dynamics of the flow around two identical circular cylinders in side-by-side arrangement has been investigated by both experiments and numerical simulations. The center-to-center transverse pitch ratio $T / D$ plays an important role in determining the flow features. It is observed that for $1<T / D<$ 1.1 to 1.2 , a single vortex street is formed; for $1.2<T / D<2$ to 2.2 , bi-stable narrow and wide wakes are formed; for $2.7<T / D<4$ or 5 , anti-phase or in-phase vortex streets are formed. In the current study, the vortex structures of turbulent flows past two slightly heated side-by-side circular cylinders are investigated employing the large eddy simulation (LES). Simulations are performed using a commercial CFD software, FLUENT. The Smagorinsky-Lilly subgrid-scale model is employed for the large eddy simulation. The Reynolds number based on free-stream velocity and cylinder diameter is 5800 , which is in the subcritical regime. The transverse pitch ratio $T / D=3$ is investigated. Laminar boundary layer, transition in shear layer, flow separation, large vortex structures and flow interference in the wake are all involved in the flow. Such complex flow features make the current study a challenging task. Both flow field and temperature field are investigated. The calculated results are analyzed and compared with experimental data. The simulation results are qualitatively in accordance with experimental observations. Two anti-phase vortex streets are obtained by the large-eddy simulation, which agrees with the experimental observation. At this transverse pitch ratio, these two cylinders behave as independent, isolated single cylinder in cross flow. The time-averaged streamwise velocity and temperature at $x / D=10$ are in good agreement with the experimental data. Figure1 displays the instantaneous spanwise vorticity at the center plane.
\end{abstract}

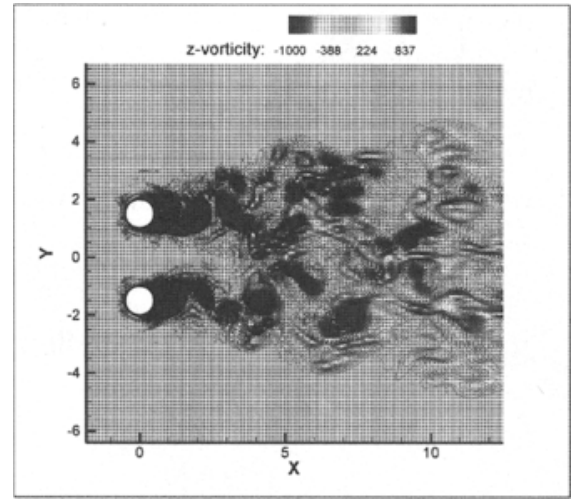

Figure 1: Instantaneous spanwise vorticity at the center plane 